\title{
Forensic and medico-legal radiology: challenges, issues and new perspectives
}

\author{
Giuseppe Guglielmi $^{1,2} \cdot$ Michelangelo Nasuto $^{1} \cdot$ Antonio Pinto $^{3}$
}

Published online: 2 August 2015

(C) Italian Society of Medical Radiology 2015

During the last decades the radiologist's world has been significantly influenced by the waves of technological progress. Higher anatomical detail and multi-parametric analysis of pathological changes have widened the imaging spectrum and set new health quality standards demanding a more complex professional figure.

Medico-legal radiology has thus reflected this evolution through a dichotomous path: one bridging radiology with forensic medicine, and the other focusing on issues and malpractice lawsuits.

Both sides of this evolution rely in this special feature, which encloses the contributions of international experts in the fields of forensic radiology and legal medicine with the aim to provide a comprehensive overview on "the state of the art" and new perspectives on future developments.

The radiologist's deeper involvement in the diagnostic and clinical management of the patient revealed a duality of new, attractive challenges versus growing duties, liabilities, higher risks, and more sources of diagnostic errors. Moreover, the evolution of case law [1] and the growing awareness of healthcare safety have dramatically raised the legal claims and, as a consequence, the phenomenon of the "defensive medicine" [2]. Malpractice lawsuits against radiologists are commonly related to diagnostic errors, improper use of

Giuseppe Guglielmi

giuseppe.guglielmi@unifg.it

1 Department of Radiology, University of Foggia, Viale Luigi Pinto 1, 71100 Foggia, Italy

2 Department of Radiology, Scientific Institute "Casa Sollievo della Sofferenza" Hospital, Viale Cappuccini, 1 San Giovanni Rotondo, 71013 Foggia, Italy

3 Department of Radiology, “A. Cardarelli” Hospital, Naples, Italy off-label devices or contrast agents and issues with physician-patient relationship [3]. As a consequence, radiologists have to be aware of the most common errors in observation, interpretation and communication [4]. Even the increasing use of contrast media requires extensive knowledge in terms of use appropriateness, choice of the right contrast agent, management of the emergency scenarios and providing proper informed consent to the patient.

An adequate understanding of these main medico-legal issues could positively influence the radiologist's clinical routine and the behaviour of patients and referring physicians, leading towards a significant reduction of errors and malpractice legal claims.

On the other side, forensic radiology has arisen from two disciplines both based on the interpretation of anatomical-pathological findings. Before the advent of multi detector computed tomography (MDCT), the "forensic role" of radiology remained substantially circumscribed to the identification of foreign bodies (bullets above all) by conventional radiology [5].

Nowadays the improvements in cross-sectional imaging and post-processing techniques have ensured that MDCT plays a key-role in the emergency assessment of hemodynamically stable patients with penetrating wounds. The identification of wound track, its extension and its relationships with the surrounding organs are crucial for the therapeutic choice and can be very challenging for the forensic radiologist, who is supposed to know the different patterns of injuries and even the related legal aspects.

Besides the "in vivo" setting, the true revolution in forensic radiology has come for post-mortem imaging, which for decades constituted a marginal, ancillary technique of autopsy [6]. In addition to the identification of foreign bodies, a comparison of ante-mortem with post-mortem radiographs was adopted since the 1930s for the crosscheck of 
unique anatomical details and, together with dental X-rays, is still considered as an effective technique for human identification [7]. To this regard, a recent field of research called "Virtual Anthropology" merges X-Ray, ultrasound, MDCT, and magnetic resonance imaging (MRI) with anthropological criteria in order to estimate age, sex, geographical origin and stature in living and (above all) dead people $[8,9]$.

The introduction of MDCT in post-mortem imaging had a huge impact on the forensic field, due to its three-dimensional and multi-parametrical acquisition of the state of the cadaver before the irreversible changes made by autopsy and thanatological processes. Multiple (eventually remote) re-evaluations and advanced post-processing techniques allowed by digital data collection have enhanced the role of post-mortem MDCT (PMCT) as useful procedure to be conducted before the autopsy [10]. The detailed visualisation of bone structures and the detection of internal haemorrhage and foreign bodies in locations less accessible to conventional techniques of examination, revealed MDCT to be particularly suitable for the diagnosis and identification of homicide and disaster victims [11]. However, poor soft tissue contrast and weak visualisation of vessels has limited its diagnostic value to major vascular lesions.

During the last years, the idea of a contrast-enhanced PMCT angiography (PMCTA) capable of improving the diagnostic performances in coronary heart disease, pulmonary embolism and other vascular pathologies has become a target of several centres worldwide [12]. The absence of blood flow and the post-mortem changes of the vascular system such as blood clots and augmented permeability of vessel walls led to the proposal of various injection and circulation techniques with specific mixtures of contrast agents [13]. Although the scattered distribution on the territory and the different degree of validation among all the approaches, in some centres PMCTA has become a routine investigation showing promising results in comparison with conventional autopsy, particularly in the detection of the source of bleeding and coronary occlusions/stenosis $[14,15]$.

Despite the impressive advances highlighted in this collection, the complexity of this emerging subspecialty still requires intensive research, standardisation, and accreditation by the international communities. The inhomogeneous experiences and interactions with the different law systems represent a major challenge for radiological and forensic organisations. Most of all, post-mortem imaging requires extensive and specific training: the forensic radiologist must know the strengths and weakness of each imaging technique, being aware of pitfalls and artefacts typical of post-mortem changes.

We hope that this volume will give a useful, accurate focus on the main legal aspects of radiological clinical practice and will provide new perspectives on future challenges and developments in forensic radiology.

\section{Compliance with ethical standards}

Conflict of interest The authors declare that they have no conflict of interest.

Ethical standards This article does not contain any studies with human participants or animals performed by any of the authors.

\section{References}

1. Waxman DA, Greenberg MD, Ridgely MS, Kellermann AL, Heaton P (2014) The effect of malpractice reform on emergency department care. N Engl J Med 371(16):1518-1525. doi:10.1056/NEJMsa1313308

2. Hendee WR, Becker GJ, Borgstede JP, Bosma J, Casarella WJ, Erickson BA, Maynard CD, Thrall JH, Wallner PE (2010) Addressing overutilization in medical imaging. Radiology 257(1):240-245. doi:10.1148/radiol.10100063

3. Fileni A, Magnavita N, Mirk P, Iavicoli I, Magnavita G, Bergamaschi A (2010) Radiologic malpractice litigation risk in Italy: an observational study over a 14-year period. AJR Am J Roentgenol 194(4):1040-1046. doi:10.2214/AJR.09.3457

4. Berlin L (2013) Medicolegal-malpractice and ethical issues in radiology: reporting the "missed" radiologic diagnosis. AJR Am J Roentgenol 201(3):W516. doi:10.2214/AJR.13.10759

5. Pomara C, Fineschi V, Scalzo G, Guglielmi G (2009) Virtopsy versus digital autopsy: virtual autopsy. Radiol Med 114(8):13671382. doi:10.1007/s11547-009-0435-1

6. Guglielmi G, Nasuto M (2014) Forensic radiology special feature: preface. Br J Radiol 1036:20140153. doi:10.1259/bjr.20140153

7. Culbert WC, Law FM (1927) Identification by comparison of roentgenograms of nasal accessory sinuses and mastoid process. J Am Med Assoc 88(4):1634-1636

8. Dedouit F, Savall F, Mokrane FZ, Rousseau H, Crubezy E, Rouge D et al (2014) Virtual anthropology and forensic identification using multidetector CT. Br J Radiol 87:20130468

9. Dedouit F, Guglielmi G, Perilli G, Nasuto M, Telmon N, Fineschi V, Pomara C (2014) Virtual anthropological study of the skeletal remains of San Fortunato (Italy, third century AD) with multislice computed tomography. JOFRI 2:9-16. doi:10.1016/j. jofri.2013.11.006

10. Roberts IS, Benamore RE, Benbow Jackson A, Mallett S, Patankar T, Peebles C, Roobottom C, Traill ZC (2012) Postmortem imaging as an alternative to autopsy in the diagnosis of adult deaths: a validation study. Lancet 379:136-142

11. Baglivo M, Winklhofer S, Hatch GM, Ampanozi G, Thali MJ, Ruder TD (2013) The rise of forensic and postmortem radiology —analysis of the literature between the year 2000 and 2011. J Forensic Radiol Imag 1(1):3-9

12. Morgan B, Biggs MJ, Barber J, Raj V, Amoroso J, Hollingbury FE, Robinson C, Rutty GN (2013) Accuracy of targeted post- mortem computed tomography coronary angiography compared to assessment of serial histological sections. Int J Leg Med 127:809-817

13. Pomara C, Bello S, Grilli G, Guglielmi G, Turillazzi E (2015) Multi-phase postmortem CT angiography (MPMCTA): a new axillary approach suitable in fatal thromboembolism. Radiol Med 120(7):670-673. doi:10.1007/s11547-014-0467-z

14. Grabherr S, Grimm J, Dominguez A, Vanhaebost J, Mangin P (2014) Advances in post-mortem CT-angiography. Br J Radiol 87(1036):20130488. doi:10.1259/bjr.20130488

15. Ross SG, Bolliger SA, Ampanozi G, Oesterhelweg L, Thali MJ, Flach PM (2014) Postmortem CT angiography: capabilities and limitations in traumatic and natural causes of death. Radiographics 34(3):830-846. doi:10.1148/rg.343115169 